\title{
Surface Evolution Under Curvature Flows
}

\section{Citation}

Lu, Conglin, Yan Cao, and David B. Mumford. 2002. Surface evolution under curvature flows. Journal of Visual Communication and Image Representation 13(1-2): 65-81.

\section{Published Version}

doi:10.1006/jvci.2001.0476

\section{Permanent link}

http://nrs.harvard.edu/urn-3:HUL.InstRepos:4726298

\section{Terms of Use}

This article was downloaded from Harvard University's DASH repository, and is made available under the terms and conditions applicable to Other Posted Material, as set forth at http:// nrs.harvard.edu/urn-3:HUL.InstRepos:dash.current.terms-of-use\#LAA

\section{Share Your Story}

The Harvard community has made this article openly available.

Please share how this access benefits you. Submit a story.

Accessibility 


\title{
Surface Evolution under Curvature Flows
}

\author{
Conglin Lu, Yan Cao, David Mumford \\ Division of Applied Mathematics, Brown University, Providence, RI 02912 \\ E-mail: clu@cfm.brown.edu; cao@cfm.brown.edu; David_Mumford@brown.edu
}

Submitted for the special issue on Partial Differential Equations (PDE's)

in Image Processing, Computer Vision, and Computer Graphics.

\section{Correspondence:}

Conglin Lu

Division of Applied Mathematics

Brown University, Box F

Providence, RI 02912

Email: clu@cfm.brown.edu

Phone: (401) 863-2261

Fax: (401) 863-1355 
In many areas of computer vision, such as multiscale analysis and shape description, an image or surface is smoothed by a non-linear parabolic partial differential equation to eliminate noise and to reveal the large global features. An ideal flow, or smoothing process, should not create new features. In this paper we describe in detail the effect of a number of flows on surfaces on the parabolic sets, the ridge curves and umbilic points. In particular we look at the mean curvature flow and the two principal curvature flows. Our calculations show that two principal curvature flows never create parabolic and ridge curves of the same type as the flow, but no flow is found capable of simultaneously smoothing out all features. In fact, we find that the principal curvature flows in some cases create a highly degenerate type of umbilic. We illustrate the effect of these flows by an example of a 3-D face evolving under principal curvature flows.

Key Words: Curvature flow, parabolic curve, ridge curve, umbilic point 


\section{INTRODUCTION}

Geometry-driven flows have been proposed and studied for smoothing surfaces or getting a hierarchical description of surfaces. It is believed that shape features are only meaningful over a particular range of scale [1]. Some features are pure noise associated to the measurement process, some are fine details (think of wrinkles on a face), some are of intermediate scale and some are present on the coarsest scale (think of the nose as a feature of facial shape). Therefore, in order to describe a shape, it is extremely important to get a multiscale representation of it. This basic idea led to the development of scale-space theory [2,3]. Initially the work was focused only on linear scale-spaces, but later many non-linear and geometric scale-space methods were also developed (for example, the anisotropic diffusion proposed by Perona et al. [4, 5], the level set method described in Osher-Sethian [6], and Olver et al.'s work based on differential invariants [7]. See also Brakke [8], Gage-Hamilton [9], Alvarez-Lions-Morel [10], etc.)

Descriptions of a shape at different scales are obtained by continuously deforming it to smoother ones. Ideally, this deformation process should be causal [1], in the sense that it should maintain a hierachical structure of geometric features and not introduce any new ones. Usually, these features can be characterized as certain types of singularities of a shape. The simplest example of a causal flow is given by the smoothing of a plane curve by its curvature. The main features of a plane curve are its points of inflection where the curvature is zero, and the 'vertices' where the curvature has a local maximum or minimum. Under curvature flows, these features are never created. For surfaces there are two principal curvatures and the features we will be interested in are (a) the parabolic curves where one of these curvatures is zero, (b) the ridge curves where one of them has a maximum or minimum on its corresponding line of curvature, and (c) umbilic points where they are equal.

Parabolic points are associated with inflections on object contours. It has also been shown that they are closely related to pairs of specular points on surfaces[1]. Ridge curves are highly significant features of a surface for shape recognition and analysis as they correspond roughly to what we perceive as the convex and concave 'edges' of a shape. Recent applications include medical imaging, for example the description of the cortical surface in MRI scans using ridges [11, 12], and face recognition by ridges $[13,14]$, among others. The last reference also contains a detailed exposition of the basic definitions and properties of these special curves and points on surfaces.

We will consider the class of curvature flows based on functions of the two principal curvatures. In particular we would like to know whether a certain flow creates those singular sets (features). In a rather discouraging article 10 years ago, Yuille [15] showed that both parabolic curves and ridge curves can be created under mean curvature flows. However, each parabolic and each ridge curve is associated to only one of the two principal curvatures. This suggests studying flows that can decouple the two. Our main result is that if we use the flow defined by one of the principal curvatures, then ridges and parabolics associated to that principal curvature are never created. However, umbilics are precisely those points where the two principal curvatures become equal, hence they intertwine the two flows. In fact, surfaces apparently become non- $C^{2}$ at umbilic points under principal curvature flow, this flow 
existing only as a viscosity solution. We find that umbilics can be created under both these flows, and, in fact, in some cases, umbilic points collide in a remarkable way under the principal curvature flows. Presumably, this is connected to the fact that on the simplest of all surface, namely the sphere, all points are umbilics. Moreover, our numerical experiments strongly suggest that all parabolics will eventually be eliminated under the principal curvature flows and the ridges reduced to the bare minimum (e.g. on all ellipsoid, there are three ridges on the three coordinate planes). This means that these flows are basic tools to use in connection with the application of curvature to three-dimensional object recognition.

This paper proceeds as follows. Section 2 derives the equations characterizing the flows we will consider, and includes a discussion on the existence of solutions of the equations. Sections 3 through 5 describe the local effects of curvature flows on parabolic points, ridge points, and umbilics, respectively. Section 6 shows a numerical simulation of a $3-\mathrm{D}$ face under two principal curvature flows, and gives some intuition on how the two flows simplify a surface in different ways.

\section{CURVATURE FLOW ON A MONGE PATCH}

\subsection{The Equation for Curvature Flows}

In this paper, we shall consider a smooth surface patch locally described in Monge form:

$$
\begin{aligned}
z= & f(x, y) \\
= & \frac{1}{2}\left(\kappa_{1} x^{2}+\kappa_{2} y^{2}\right)+\frac{1}{3 !} \sum_{j=0}^{3}\left(\begin{array}{l}
3 \\
j
\end{array}\right) b_{j} x^{3-j} y^{j} \\
& +\frac{1}{4 !} \sum_{j=0}^{4}\left(\begin{array}{l}
4 \\
j
\end{array}\right) c_{j} x^{4-j} y^{j}+\frac{1}{5 !} \sum_{j=0}^{5}\left(\begin{array}{l}
5 \\
j
\end{array}\right) d_{j} x^{5-j} y^{j}+o\left((x, y)^{5}\right)
\end{aligned}
$$

At the origin, the tangent plane is the $x-y$ plane, $\kappa_{1}, \kappa_{2}$ are the two principal curvatures, and the $x$ - and $y$-axes are in the principal directions (provided $\kappa_{1} \neq$ $\kappa_{2}$ ). After a suitable translation and rotation, any point on the surface can be represented this way. Without loss of generality, we always assume that $\kappa_{1} \geq \kappa_{2}$.

In the following sections we will only look at a small neighborhood of the origin. In each case we shall assume that the origin is of the singularity type in which we are interested. Then we will analyze how the features change locally by examining the corresponding equations, in terms of the coefficients of Eq. (1).

Now consider a one-parameter family of surfaces $\left\{\mathcal{S}_{t}\right\}$ parametrized by $t$, which is often referred to as 'time' or 'scale'. At time $t$, we want to deform $\mathcal{S}_{t}$ along the normal direction of each point, with a 'speed' of $\beta$ :

$$
\frac{d \mathcal{S}_{t}}{d t}=\beta \overrightarrow{\mathcal{N}_{t}}
$$

Locally, $\mathcal{S}_{t}$ can be represented in Monge form as

$$
\mathcal{S}_{t}=\{(x, y, z): z=F(x, y, t)\},
$$


and the normal vector $\overrightarrow{\mathcal{N}}_{t}$ on $\mathcal{S}_{t}$ is

$$
\overrightarrow{\mathcal{N}}_{t}=\frac{\left(-F_{x},-F_{y}, 1\right)}{\sqrt{1+F_{x}^{2}+F_{y}^{2}}}
$$

Using first order approximation, Eq. (2) becomes

$$
\mathcal{S}_{t+\Delta t}-\mathcal{S}_{t}=(\Delta x, \Delta y, \Delta z)=\beta \frac{\left(-F_{x},-F_{y}, 1\right)}{\sqrt{1+F_{x}^{2}+F_{y}^{2}}} \cdot \Delta t+o(\Delta t),
$$

where

$$
\Delta z=\Delta F=F_{x} \Delta x+F_{y} \Delta y+F_{t} \Delta t+o(\Delta t)
$$

Therefore

$$
\begin{aligned}
F_{t} & =\left(\frac{\beta \Delta t}{\sqrt{1+F_{x}^{2}+F_{y}^{2}}}-F_{x} \Delta x-F_{y} \Delta y\right) / \Delta t+o(\Delta t) / \Delta t \\
& =\beta \sqrt{1+F_{x}^{2}+F_{y}^{2}} . \quad(\text { as } \Delta t \rightarrow 0)
\end{aligned}
$$

If we suppose $\mathcal{S}_{0}$ is described by $z=f(x, y)$, as in Eq. (1), then Eq. (2) leads to the following initial value problem:

$$
\begin{aligned}
& F_{t}(x, y, t)=\beta \cdot \sqrt{1+F_{x}^{2}+F_{y}^{2}} \\
& F(x, y, 0)=f(x, y)
\end{aligned}
$$

For small $t$, we can approximate the solution by

$$
\begin{aligned}
F(x, y, t) & =F(x, y, 0)+F_{t}(x, y, 0) t+o(t) \\
& =f(x, y)+\beta \sqrt{1+f_{x}^{2}+f_{y}^{2}} \cdot t+o(t)
\end{aligned}
$$

If $\beta$ is chosen to be a function of the principal curvatures $K_{1}(x, y), K_{2}(x, y)$ (by convention, we always assume $K_{1} \geq K_{2}$ ), then we call this process of deformation a curvature flow. One of the most important types of flows is the mean curvature flow, i.e., when $\beta=H$, where $H$ is the mean curvature of the surface. The family of surfaces are defined by

$$
F(x, y, t)=f(x, y)+H(x, y) \sqrt{1+f_{x}^{2}+f_{y}^{2}} \cdot t+o(t)
$$

We will also explore principal curvature flows: when $\beta$ is one of the two principal curvatures. If $\beta=K_{1}$ (resp. $K_{2}$ ) we call the corresponding flow $K_{1}$-flow (resp. $K_{2}$-flow). Under $K_{i}$-flow $(i=1,2)$, the family of surfaces are then given by

$$
F(x, y, t)=f(x, y)+K_{i}(x, y) \sqrt{1+f_{x}^{2}+f_{y}^{2}} \cdot t+o(t)
$$


Eq. (2) is a nonlinear parabolic PDE. Even when the initial surface is smooth, the evolution family of surfaces may develop singularities. In particular, the principal curvatures are non-differentiable functions at umbilic points, hence umbilics will become singular points under the principal curvature flows. The issue of the existence and uniqueness of solution to (2) is not trivial. See Appendix for a discussion on this.

\subsection{Good Flows vs. Bad Flows}

A 'good' flow should always simplify a surface. That is, as $t$ increases, no new geometric features or singularities should be generated. Analogously, as the scale $t$ increases, no new detail is created on the surface. In fact, features should eventually be destroyed. This monotonic decrease of features is desirable because it gives us a good hierarchical description of the surface.

First, let's look at the analogous 2-D case. Consider the flow

$$
\frac{d \mathcal{C}_{t}}{d t}=\kappa \overrightarrow{\mathcal{N}}_{t}
$$

where $\left\{\mathcal{C}_{t}=(x, F(x, t))\right\}$ is a family of curves, $\kappa$ is the curvature and $\overrightarrow{\mathcal{N}}_{t}$ is the normal direction of $\mathcal{C}_{t}$. One can show that the flow leads to the equation

$$
F_{t}=F_{x x} /\left(1+F_{x}^{2}\right)
$$

As an example, consider the curve $y=x^{4}$, which has a double inflection point at $(0,0)$. One can derive

$$
\begin{aligned}
F(x, t) & =x^{4}+12 x^{2} t+o\left(x^{4}, t x^{2}\right) \\
\frac{\partial^{2} F}{\partial x^{2}}=0 & \Longleftrightarrow 12\left(x^{2}+2 t\right)+o\left(t, x^{2}\right)=0
\end{aligned}
$$

Thus there are two inflection points if $t<0$ and none for $t>0$, i.e. the flow does not create inflection points. Similarly, the flow does not create 'vertices', where the curvature assumes extremal values. Thus, this flow has the desired property.

In the 3 - $\mathrm{D}$ case, the important types of singular points include parabolic points, ridge points, umbilic points and cusps of Gauss. Again, good flows are those which do not generate new such singular points on the surface.

There is a standard bifurcation for the birth/death of each type of singularities. We will adopt the terminology in Bruce-Giblin-Tari $[16,17]$ to refer to these bifurcations. We shall examine in which direction each bifurcation moves, under different types of flows.

Remark. There are two points we'd like to emphasize here. Firstly, the results we state in this paper are local ones, concerning surface patches rather than closed surfaces. Secondly, unless otherwise mentioned, we will focus on generic surfaces, for which the features occur and change in a stable way, i.e., if we slightly perturb the surface the pattern in which the features evolve doesn't change. In contrast, surfaces of revolution are not generic, because the symmetries would be broken by small perturbations. 


\section{PARABOLIC POINTS}

At parabolic points the Gaussian curvature of a surface vanishes. They are the boundaries between elliptic and hyperbolic regions. Alternatively, they are the points where the tangent planes have a specially higher order contact with the surface [16]. Parabolic points can be further classified distinction by assigning 'colors' to them as follows: a point is called a blue parabolic point if the larger principal curvature $\kappa_{1}$ is 0 ; likewise, a red parabolic point is where the smaller principal curvature $\kappa_{2}$ equals 0 . A more degenerate type is the flat umbilic, where $\kappa_{1}=\kappa_{2}=0$.

If the surface is closed and oriented so that the curvature is positive at convex regions, then the red parabolics are the boundaries between convex elliptic regions and hyperbolic regions and the blue parabolics are the boundaries of the concave elliptic regions.

Generically, in a one parameter family of surfaces, parabolic curves can only be created through a non-versal $A_{3}$ transition [16]. At the moment of transition there is (locally) a single parabolic point. Then it either disappears or evolves into a parabolic loop.

The red parabolic set (i.e., $K_{2}=0$ ) of $\mathcal{S}_{0}$ around the origin satisfies

$$
\begin{aligned}
0= & f_{x x} f_{y y}-f_{x y}^{2} \\
= & \kappa_{1}\left(b_{2} x+b_{3} y\right)+\left(\frac{1}{2} \kappa_{1} c_{2}+b_{0} b_{2}-b_{1}^{2}\right) x^{2} \\
& +\left(\kappa_{1} c_{3}+b_{0} b_{3}-b_{1} b_{2}\right) x y+\left(\frac{1}{2} \kappa_{1} c_{4}+b_{1} b_{3}-b_{2}^{2}\right) y^{2} \\
& +o\left((x, y)^{2}\right) .
\end{aligned}
$$

If a red parabolic loop is to be created, then there must exist a moment when an $A_{3}$ transition takes place at some point on the surface. For the rest of this section, we will suppose that $t=0$ corresponds to the moment of an $A_{3}$ transition; and that the origin is an isolated red parabolic point on $\mathcal{S}_{0}$. Thus $b_{2}=b_{3}=0$, and the quadratic form

$$
Q(x, y)=\left(\frac{1}{2} \kappa_{1} c_{2}-b_{1}^{2}\right) x^{2}+\kappa_{1} c_{3} x y+\frac{1}{2} \kappa_{1} c_{4} y^{2}
$$

is either positive definite or negative definite in a neighborhood of the origin.

\subsection{Mean Curvature Flow}

Under mean curvature flows, the family of surfaces (for small $t$ ) is given by Eq. (5). The parabolic set of $\mathcal{S}_{t}$ is given by

$$
\begin{aligned}
0= & F_{x x} F_{y y}-F_{x y}^{2} \\
= & {\left[\frac{1}{2} \kappa_{1}\left(c_{2}+c_{4}\right)+O(x, y)\right] t+o(t) } \\
& +\left(\frac{1}{2} \kappa_{1} c_{2}-b_{1}^{2}\right) x^{2}+\kappa_{1} c_{3} x y+\frac{1}{2} \kappa_{1} c_{4} y^{2}+o\left((x, y)^{2}\right) .
\end{aligned}
$$


For each fixed $t$, the parabolic set satisfies

$$
t=-\frac{Q(x, y)}{\kappa_{1}\left(c_{2}+c_{4}\right) / 2}+o\left((x, y)^{2}\right)
$$

Here $Q(x, y)$ is either positive definite or negative definite, depending on the sign of $\kappa_{1} c_{4}$ :

- If $\kappa_{1} c_{4}>0$, then $\frac{1}{2} \kappa_{1} c_{2}-b_{1}^{2}>0$, so that $\kappa_{1} c_{2}>0$. Hence, $\kappa_{1}\left(c_{2}+c_{4}\right)>0$, and the right hand side of $(7)$ is always negative. Thus for sufficiently small $t>0$, no red parabolic loop is created;

- If $\kappa_{1} c_{4}<0$, it is possible to create red parabolic loops for some surfaces, since the right hand side of $(7)$ could be positive.

The case $\kappa_{1} c_{4}>0$ corresponds to the bifurcation when a hyperbolic area appears or disappears inside an elliptic region; $\kappa_{1} c_{4}<0$ corresponds to the opposite case[16]. Thus, no hyperbolic regions can be created under a mean curvature flow. However, elliptic regions can be created. By symmetry, this is also true when we start from an isolated blue parabolic point.

\subsection{Principal Curvature Flow}

Now consider the $K_{2}$-flow. Recall that $\kappa_{2}=0$. The smaller principal curvature around the origin is

$$
K_{2}(x, y)=\left(\frac{c_{2}}{2}-\frac{b_{1}^{2}}{\kappa_{1}}\right) x^{2}+c_{3} x y+\frac{c_{4}}{2} y^{2}+o\left((x, y)^{3}\right)
$$

Substituting this into (6), we can obtain $F(x, y, t)$, and the parabolic set of $\mathcal{S}_{t}$ is given by:

$$
\begin{gathered}
0=F_{x x} F_{y y}-F_{x y}^{2} \\
=\left[\frac{1}{2} \kappa_{1} c_{4}+O(x, y)\right] t+Q(x, y)+o\left(t,(x, y)^{2}\right) \\
t=-\frac{\left(\frac{1}{2} \kappa_{1} c_{2}-b_{1}^{2}\right) x^{2}+\kappa_{1} c_{3} x y+\frac{1}{2} \kappa_{1} c_{4} y^{2}}{\kappa_{1} c_{4}}+o\left((x, y)^{2}\right)
\end{gathered}
$$

The numerator and the denominator above always have the same sign, which means that for $t>0$ and small, the isolated red parabolic point is always eliminated, and no (red) parabolic loop is created. A similar argument shows that $K_{1}$-flows $d o$ not generate blue parabolic loops.

In addition, we believe that the blue (resp. red) parabolic loops are always eliminated by $K_{1}$-flows (resp. $K_{2}$-flows), although a rigorous proof has yet to be found.

\subsection{Conclusion}

- Under mean curvature flows, parabolic loops (either blue or red) can be created. More precisely, hyperbolic regions cannot be created inside elliptic regions; elliptic regions can be generated inside hyperbolic regions. 
- Under $K_{1^{-}}$(resp. $K_{2^{-}}$) flows, no blue (resp. red) parabolic loops are generated.

\section{RIDGE POINTS}

A ridge point is a point where the surface has a higher order contact with one of the osculating spheres; or equivalently, where the principal curvature has an extreme value along the corresponding line of curvature. Ridge points can also be colored: those associated with the larger principal curvature are blue ridges, and those associated with the smaller principal curvature are red ridges. Here we shall restrict ourselves to ridge points away from umbilics.

Let $K_{2}(x, y)$ be the smaller principal curvature, and $\vec{V}_{2}(x, y)$ be the principal direction corresponding to $K_{2}$. Then the condition for a red ridge point is

$$
\nabla K_{2}(x, y) \cdot \vec{V}_{2}(x, y)=0
$$

From this we can get the equation for red ridge points (in a sufficiently small neighborhood of the origin):

$$
\begin{aligned}
0= & b_{3}\left(\kappa_{1}-\kappa_{2}\right)-\left(3 b_{1} b_{2}-\left(\kappa_{1}-\kappa_{2}\right) c_{3}\right) x \\
& -\left(3 b_{2}^{2}-\left(\kappa_{1}-\kappa_{2}\right)\left(c_{4}-3 \kappa_{2}^{3}\right)\right) y \\
& +Q(x, y)+o\left((x, y)^{2}\right)
\end{aligned}
$$

where

$$
\begin{aligned}
Q(x, y)= & (\cdots) x^{2}+(\cdots) x y+\left(\frac{1}{2} d_{5}\left(\kappa_{1}-\kappa_{2}\right)-\frac{9}{2} b_{2} c_{3}\right. \\
& \left.+\frac{6 b_{1} b_{2}^{2}}{\kappa_{1}-\kappa_{2}}-9\left(\kappa_{1}-\kappa_{2}\right) \kappa_{2}^{2} b_{3}-\frac{4 b_{2}^{2} b_{3}}{\kappa_{1}-\kappa_{2}}\right) y^{2}
\end{aligned}
$$

represents the quadratic terms.

Generically, in a family of surfaces, ridges are created or killed through a Morsetransition [17]. At the moment of transition there is (locally) an isolated ridge point. Then this point either disappears or develops into a ridge loop.

Suppose that $\mathcal{S}_{0}$ is the surface at the transition moment; and that the origin is an isolated red ridge point. Then the linear terms in Eq. (8) vanish, which yields

$$
b_{3}=0,3 b_{1} b_{2}=\left(\kappa_{1}-\kappa_{2}\right) c_{3}, 3 b_{2}^{2}=\left(\kappa_{1}-\kappa_{2}\right)\left(c_{4}-3 \kappa_{2}^{3}\right)
$$

Eq. (8) now reduces to

$$
Q(x, y)+o\left((x, y)^{2}\right)=0
$$

with

$$
\begin{aligned}
Q(x, y)= & (\cdots) x^{2}+(\cdots) x y \\
& +\frac{1}{2}\left(d_{5}\left(\kappa_{1}-\kappa_{2}\right)-\frac{15 b_{1} b_{2}^{2}}{\kappa_{1}-\kappa_{2}}\right) y^{2}
\end{aligned}
$$


being either positive definite or negative definite in a neighborhood of the origin.

\subsection{Mean Curvature Flow}

It can be shown that the mean curvature flow can move in either direction; i.e., it can either generate a ridge loop or not. The calculations, which are omitted here, are similar to those in the following subsection.

\subsection{Principal Curvature Flow}

The equation for the red ridge curve on $\mathcal{S}_{t}$ is described by

$$
\nabla K_{2}(x, y, t) \cdot \vec{V}_{2}(x, y, t)=0
$$

which leads to

$$
\begin{aligned}
0= & Q(x, y)+\left(d_{5}\left(\kappa_{1}-\kappa_{2}\right)-\frac{15 b_{1} b_{2}^{2}}{\kappa_{1}-\kappa_{2}}\right) t \\
& +o\left(t,(x, y)^{2}\right) .
\end{aligned}
$$

Solving for $t$ we get

$$
t=-\frac{Q(x, y)}{d_{5}\left(\kappa_{1}-\kappa_{2}\right)-\frac{15 b_{1} b_{2}^{2}}{\kappa_{1}-\kappa_{2}}}+o\left((x, y)^{2}\right) .
$$

Notice that the denominator and the coefficient of $y^{2}$ in $Q(x, y)$ always have the same signs. By assumption, locally $Q(x, y)$ is either positive definite or negative definite. Thus $t$ would always be negative when $x$ and $y$ are small. This means that when $t>0$ there is no ridge point, and therefore the $K_{2}$-flow cannot generate any red ridges. By a similar argument, we can prove that $K_{1}$-flows do not generate any blue ridges.

In conclusion, $K_{1^{-}}$(resp. $K_{2^{-}}$) flows do not generate blue (resp. red) ridge loops. As in the parabolic case, we suspect that these flows always eliminate the ridge loops of the corresponding color.

\section{UMBILICS}

At an umbilic point the two principal curvatures are equal. The ridge curves change 'colors' at umbilic points.

When a family of surfaces goes through a non-versal $D_{4}$ transition, a pair of umbilics are either created or killed [17]. Suppose at the moment of transition, the origin is a (double) umbilic point on $\mathcal{S}_{0}$. One can show that $\mathcal{S}_{0}$ is given by

$$
\begin{aligned}
z= & f(x, y) \\
= & \frac{1}{2} \kappa\left(x^{2}+y^{2}\right)+\frac{1}{6}\left(b_{0} x^{3}+3 b_{1} x^{2} y+3 b_{2} x y^{2}+b_{3} y^{3}\right) \\
& +o\left((x, y)^{3}\right),
\end{aligned}
$$

with the coefficients satisfying

$$
\left|\begin{array}{cc}
b_{0}-b_{2} & b_{1}-b_{3} \\
b_{1} & b_{2}
\end{array}\right|=0
$$


By rotating axes we can assume $b_{1}=b_{2}=0$. We will consider several different types of flows in this section.

\subsection{Modified Mean Curvature Flow}

First consider the modified mean curvature flow

$$
F(x, y, t)=f(x, y)+H_{n}(x, y) \sqrt{1+f_{x}^{2}+f_{y}^{2}} \cdot t+o(t)
$$

where

$$
H_{n}(x, y)=\left(\frac{K_{1}^{n}(x, y)+K_{2}^{n}(x, y)}{2}\right)^{1 / n} .
$$

Here $K_{1}(x, y), K_{2}(x, y)$ are the two principal curvatures.

$H_{n}(x, y)$ is actually a function of the mean curvature $H(x, y)$ and the Gaussian curvature $K(x, y)$. From the Taylor expansion of $H$ and $K$, we can get the expansion of $H_{n}$ around the origin. The details are omitted here. The result is

$$
\begin{aligned}
H_{n}= & \kappa+\frac{1}{2}\left(b_{0} x+b_{3} y\right)+ \\
& \left(-\kappa^{3}+\frac{1}{4} c_{0}+\frac{1}{4} c_{2}+\frac{n-1}{8} \frac{b_{0}^{2}}{\kappa}\right) x^{2}+ \\
& \left(\frac{1}{2} c_{1}+\frac{1}{2} c_{3}-\frac{n-1}{4} \frac{b_{0} b_{3}}{\kappa}\right) x y+ \\
& \left(-\kappa^{3}+\frac{1}{4} c_{2}+\frac{1}{4} c_{4}+\frac{n-1}{8} \frac{b_{3}^{2}}{\kappa}\right) y^{2}+o\left((x, y)^{2}\right)
\end{aligned}
$$

Suppose that the first and second fundamental forms of $\mathcal{S}_{t}$ are $I=\mathbf{E} d x^{2}+$ $2 \mathbf{F} d x d y+\mathbf{G} d y^{2}$ and $I I=\mathbf{e} d x^{2}+2 \mathbf{f} d x d y+\mathrm{g} d y^{2}$, respectively. One can show the condition for an umbilic is

$$
\operatorname{rank}\left(\begin{array}{ccc}
\mathbf{E} & \mathbf{F} & \mathbf{G} \\
\mathbf{e} & \mathbf{f} & \mathrm{g}
\end{array}\right) \leq 1
$$

which is equivalent to simultaneously requiring

$$
\begin{aligned}
& \mathbf{E} g-\mathbf{G} e=0 \\
& \mathbf{G} f-\mathbf{F} g=0
\end{aligned}
$$

Notice that we can replace $\mathbf{e}, f, g$ by $F_{x x}, F_{x y}$, and $F_{y y}$ in the above equations. We can calculate the Taylor expansions of these terms and substitute them into the equations. It turns out that (10) is a linear equation for $x$ and $y$, whereas (11) is a quadratic one. If we solve for $t$ we get

$$
t=-\frac{2 \kappa\left(2 b_{0} b_{3} c_{2}+b_{0}^{2} c_{3}+b_{3}^{2} c_{1}-2 \kappa^{3} b_{0} b_{3}\right)}{b_{3}^{2}\left(2 \kappa c_{1}+2 \kappa c_{3}-(n-1) b_{0} b_{3}\right)} x^{2}+o\left(x^{2}\right)
$$

Since for a generic surface, the right hand side of (12) could be either positive or negative, our flow can go in both directions, meaning that it can either create or kill a pair of umbilics. 
The above result is derived for $n>1$. A direct calculation shows that it also holds when $n=1$, which is the case for mean curvature flows.

\subsection{Some Other Types of Flows}

Next consider the principal curvature flows $\left(\beta=K_{1}\right.$ or $\left.\beta=K_{2}\right)$. Since the principal curvatures are not $C^{\infty}$ functions at the umbilic points, we cannot directly expand them into Taylor series. However, it is clear that the $K_{1}$-flow is the limiting case of the $H_{n}$-flow above (as $n \rightarrow \infty$ ). Also, if the $K_{1}$-flow can go in both directions, so can the $K_{2}$-flow, because they have the same effect in this case. Consequently, the principal curvature flows can go in both directions as well.

Another interesting case is the Gaussian curvature flow, when $\beta=G=K_{1} K_{2}$. By similar calculations as in section 5.1, we get

$$
t=-\frac{1}{2} \frac{2 b_{0} b_{3} c_{2}+b_{0}^{2} c_{3}+b_{3}^{2} c_{2}-2 \kappa^{3} b_{0} b_{3}}{b_{3}^{2}\left(\kappa c_{1}+\kappa c_{3}+b_{0} b_{3}\right)} x^{2}+o\left(x^{2}\right) .
$$

Finally, consider the mean-Gaussian flow proposed by Neskovic and Kimia [18], in which case

$$
\beta=\operatorname{sign}(H) \cdot \sqrt{G+|G|}
$$

The result is:

$$
t \approx-\frac{\sqrt{2} \kappa\left(2 b_{0} b_{3} c_{2}+b_{0}^{2} c_{3}+b_{3}^{2} c_{1}-2 \kappa^{3} b_{0} b_{3}\right)}{b_{3}^{2}\left(2 \kappa c_{1}+2 \kappa c_{3}+b_{0} b_{3}\right)} x^{2} .
$$

Obviously both flows can create or delete umbilics.

\subsection{Conclusion}

Under any of the above flows, a pair of umbilics can either be generated or eliminated. We think this is due to the fact that the natural limit of the smoothing process is a sphere which is one big degenerate locus of umbilic points. This suggests that destroying umbilics is not an essential part of the smoothing process. From another perspective, umbilic points are conformally invariant features, whereas all the above flows are not. P.Olver (personal communication) has determined the lowest order conformally invariant formal flow but it turns out to be a parabolic flow which is everywhere ill-posed, its second order derivatives having one positive and one negative eigenvalue. A very interesting problem is to describe the form of singular surface that viscosity solutions of the principal curvature flow generate from umbilics: we conjecture that these are some sort of "pseudo-umbilic" $C^{1}$ but not $C^{2}$ points.

\section{SIMULATING PRINCIPAL CURVATURE FLOWS ON A FACE}

The experiment is based on laser range data of the face of a young woman which has been first smoothed to eliminate noisy features. The data comes from a Cyberware scanner and is in cylindrical coordinates $r=r(z, \theta)$ describing the whole head. We only look at the face and we impose Neumann boundary conditions $\frac{\partial r}{\partial \theta}=\frac{\partial r}{\partial z}=0$ to get a well-posed boundary value problem.

To understand the experiment, you must first realize that the lines of curvature of $\kappa_{1}$, the larger curvature, tend to be horizontal. Thus, when the face is smoothed 
a great deal, it approaches an ellipsoid with $\kappa_{1}$ maximum on the nose ridge and its lines of curvature perpendicular to the nose ridge and running left and right across the face area. In a fully formed face, these lines make detours around the nose, the eyes and ends of the mouth. On the other hand, the lines of curvature of $\kappa_{2}$, the smaller curvature, tend to be vertical on the smooth parts of the face (see figures $7.19,7.21$ and 7.26 of [14]).

We expect that principal curvature flows will simplify the surface mainly in the corresponding principal directions. In fact, what happens is under the $K_{1}$-flow, the face in the horizontal direction tends to become circular while in the vertical direction it retains the original undulating curve caused by eyes, nose and mouth. On the other hand, under the $K_{2}$-flow, the face in the vertical direction tends to become flat while in the horizontal direction we have a single peak along the nose, so after some time, the face looks like a folded paper. See Fig. 1.

FIG. 1. The 3D face under curvature flows.

Both the $K_{1}$-flow and the $K_{2}$-flow kill the parabolic loops: see Figure 2 . We also found that the $K_{2}$-flow created a blue parabolic loop near the boundary of the face. Although this is certainly a new structure, it is created in a nearly flat part of the face where the cheek interacts with the Neumann boundary conditions we imposed and is not a new perceptually salient structure. Moreover, under both principal curvature flows, a pair of umbilics can be either created or eliminated. A pair of umbilics is created in Fig. 2.

FIG. 2. The evolution of parabolics, ridges and umbilics.

Another interesting phenomenon in Fig. 2 is that the pair of umbilics of the same kind always present on the tip of the nose get closer under $\kappa_{1}$ flow. One might suspect that this was an artifact of the numerical simulation (as we did) but one can give a strong heuristic argument that this really happens for pairs of symmetric double umbilics on a ridge. To make the calculation relatively simple, assume we have a surface $z=f(x, y)$ symmetric under both $(x, y) \rightarrow(-x, y)$ and $(x, y) \rightarrow(x,-y)$. This makes the $\mathrm{x}$ - and $\mathrm{y}$-axes red and blue ridges respectively on this surface. In the Monge form, only terms in $x^{2}$ and $y^{2}$ remain:

$$
\begin{aligned}
z=f(x, y)= & \frac{1}{2} \kappa_{1} x^{2}+\frac{1}{2} \kappa_{2} y^{2}+\frac{1}{24}\left(c_{0} x^{4}+6 c_{2} x^{2} y^{2}+c_{4} y^{4}\right)+ \\
& \frac{1}{6 !}\left(e_{0} x^{6}+15 e_{2} x^{4} y^{2}+15 e_{4} x^{2} y^{4}+e_{6} y^{6}\right)+\ldots
\end{aligned}
$$

Then the two principal directions are $\mathrm{x}$-axis and $\mathrm{y}$-axis. Suppose there are two umbilics near the origin on the $\mathrm{x}$-axis. We get the following condition for this to happen:

$$
c_{2}-c_{0}+2 \kappa_{1}^{3}>0
$$


In this case, the $(x, y)$ coordinates of the two umbilics are $\left( \pm \sqrt{\frac{2\left(\kappa_{1}-\kappa_{2}\right)}{c_{2}-c_{0}+2 \kappa_{1}^{3}}}+\cdots, 0\right)$. Under the $K_{1}$-flow,

$$
\begin{aligned}
& F(x, y, \Delta t) \\
= & F(x, y, 0)+F_{t}(x, y, 0) \Delta t+o(\Delta t) \\
= & f(x, y)+K_{1}(x, y) \sqrt{1+f_{x}^{2}+f_{y}^{2}} \Delta t+o(\Delta t) \\
= & \kappa_{1} \Delta t+\frac{1}{2}\left(\kappa_{1}+\left(c_{0}-2 \kappa_{1}^{3}\right) \Delta t\right) x^{2}+\frac{1}{2}\left(\kappa_{2}+c_{2} \Delta t\right) y^{2} \\
& +\frac{1}{24}\left(c_{0}+\left(e_{2}-20 c_{0} \kappa_{1}^{2}+24 \kappa_{1}^{5}\right) \Delta t\right) x^{4} \\
& +\frac{1}{4}\left(c_{2}+\left(\left(e_{4}+c_{0} \kappa_{2}^{2}+2 c_{2} \kappa_{1}^{2}-4 \kappa_{1}^{5}-4 \kappa_{1}^{4} \kappa_{2}\right)+\frac{4\left(c_{2}-\kappa_{1}^{3}\right)^{2}}{\kappa_{1}-\kappa_{2}}\right) \Delta t\right) x^{2} y^{2} \\
& +\frac{1}{24}\left(c_{4}+e_{6} \Delta t\right) y^{4}+o\left(\Delta t, x^{4}, y^{4}\right)
\end{aligned}
$$

Hence,

$$
\frac{\partial\left(\kappa_{1}-\kappa_{2}\right)}{\partial t}=c_{0}-c_{2}-2 \kappa_{1}^{3}<0
$$

which brings the umbilics closer together. One can also look at the second derivative, at the change of $C_{2}-C_{0}+2 K_{1}^{3}$ :

$$
\frac{\partial\left(C_{2}-C_{0}+2 K_{1}^{3}\right)}{\partial t}=b(x, y)+\frac{4}{\kappa_{1}-\kappa_{2}}\left(c_{2}-\kappa_{1}^{3}\right)^{2}
$$

where

$$
b(x, y)=e_{4}-e_{2}+26 c_{0} \kappa_{1}^{2}+c_{0} \kappa_{2}^{2}+2 c_{2} \kappa_{1}^{2}-40 \kappa_{1}^{5}-4 \kappa_{1}^{4} \kappa_{2}
$$

is bounded in terms of the coefficients of $z$. So, when $\kappa_{1}-\kappa_{2}$ is small enough

$$
\frac{\partial\left(C_{2}-C_{0}+2 K_{1}^{3}\right)}{\partial t}>0
$$

These two conditions suggest strongly that the two umbilics will get closer under $\kappa_{1}$ flow and eventually become one highly degenerate umbilics with index +1 or -1 depending on the type of the umbilic pair we started with.

\section{SUMMARY}

We have shown that under principal curvature flows, no parabolic loop or ridge loop of the corresponding color can be created. We believe that this clears the way for the application of curvature ideas to $3 \mathrm{D}$ object recognition. In contrast, the mean curvature flow can create all types of singularities which we have considered. We believe that what this ultimately means is that, in considering the curvature structure of a surface, one should look at it as two intertwined stories: the story told by the maximum principal curvature with its ridges, parabolics and lines of curvature and the story told by the minimum principal curvature. We have seen how, in the case of the face, these two flows undo its features in very different ways. These flows should lead to a method of extracting a curvature "portrait" for 
surfaces, generalizing part of the plane curve "portrait" due to Kimia, Tannenbaum and Zucker [25]. Such a curvature portrait would be a powerful tool for 3D object recognition.

\section{APPENDIX: EXISTENCE AND UNIQUENESS OF SOLUTIONS OF PRINCIPAL CURVATURE FLOW EQUATIONS}

A theory of "viscosity solutions" has been developed to study nonlinear second order partial differential equations such as Eq. (2) [19, 20, 21, 22]. The existence of a unique viscosity solution of mean curvature flow equation is proven in Evans [23] and Chen-Giga-Goto [24]. The latter also shows that the same result holds for a more general class of geometric, degenerate parabolic equations. We will apply this result to establish the existence of unique viscosity solutions of principal curvature flow equations.

Following the notation in [24], consider the second order parabolic equation

$$
\begin{aligned}
& u_{t}+F\left(t, \nabla u, \nabla^{2} u\right)=0, \\
& u(0, x)=a(x) \in C_{\alpha}\left(\boldsymbol{R}^{n}\right),
\end{aligned}
$$

where $u=u(t, x), x \in \boldsymbol{R}^{n}$, and for a constant $\alpha, C_{\alpha}(A)$ is defined to be the set of continuous functions $a(x)$ in $A$ such that $a-\alpha$ is compactly supported in $A$. We say that Eq. (A.1) is geometric if $F$ has a scaling invariance

$$
F(t, \lambda p, \lambda X+\sigma p \otimes p)=\lambda F(t, p, X), \quad \lambda>0, \sigma \in \boldsymbol{R},
$$

for nonzero $p \in \boldsymbol{R}^{n}$ and $X \in S^{n \times n}$, the space of $n \times n$ real symmetric matrices. $F$ is called degenerate elliptic if

$$
F(t, p, X+Y) \leq F(t, p, X) \quad \text { for } Y \geq O, Y \in S^{n \times n}
$$

where $O$ is the all-zero matrix. Theorem 6.8 in [24] is restated as follows:

Theorem A.1 (Global existence [24]). Let $T>0$. Assume that $F(t, p, X)$ is continuous in $(0, T] \times\left(\boldsymbol{R}^{n} \backslash\{0\}\right) \times S^{n \times n}$ and is geometric and degenerate elliptic, and that $F$ satisfies

$$
\begin{aligned}
& F(t, p,-I) \leq c_{-}(|p|), \\
& F(t, p, I) \geq-c_{+}(|p|), \\
& \lim _{p, X \rightarrow 0} F(t, p, X) \text { exists and is finite, }
\end{aligned}
$$

for some $c_{ \pm}(\sigma) \in C^{1}[0, \infty)$ and $c_{ \pm}(\sigma) \geq c_{0}>0$ with some constant $c_{0}$. Then for $a \in C_{\alpha}\left(\boldsymbol{R}^{n}\right)$ there is a unique viscosity solution $u_{a} \in C_{\alpha}\left([0, T] \times \boldsymbol{R}^{n}\right)$ of Eq. (A.1)-(A.2).

In order to apply the theorem, we need to regard $\left\{\mathcal{S}_{t}\right\}$ as level surfaces of some function $u$, i.e., $\mathcal{S}_{t}=\{(x, y, z): u(t, x, y, z)=0\}$. The shape operator is given by the matrix $-\left(I-\frac{\nabla u}{|\nabla u|} \otimes \frac{\nabla u}{|\nabla u|}\right) \nabla^{2} u$. It appears as an operator applied to the tangent spaces in the ambient 3 -space. 0 is one of its eigenvalues, and $\nabla u$ is the 
corresponding eigenvector. The other two eigenvectors, which correspond to the two principal directions, lie in the plane perpendicular to $\nabla u$.

For $K_{1}$-flow, Eq. (2) becomes

$$
u_{t}+F\left(\nabla u, \nabla^{2} u\right)=0
$$

with

$$
F(p, X)=- \text { larger eigenvalue of }(I-\bar{p} \otimes \bar{p}) X \text { in } p^{\perp}, \quad \bar{p}=\frac{p}{|p|} .
$$

where $I$ is the identity matrix, $p^{\perp}$ is the plane perpendicular to the vector $p$.

We can show that $F$ is geometric and degenerate elliptic. Conditions (A.5_), $\left(A .5_{+}\right)$are satisfied by choosing $c_{ \pm}(\sigma) \equiv 1$. Condition (A.6) is also easily seen to be satisfied. Therefore we have

Proposition A.1. There exist unique viscosity solutions to the principal curvature flow equations.

\section{ACKNOWLEDGMENT}

The authors would like to thank Peter Giblin and Thomas Banchoff for many helpful discussions and suggestions throughout the work.

\section{REFERENCES}

1. Koenderink, J.J., Solid Shape, MIT Press: Cambridge, 1990.

2. Witkin, A.P., "Scale space filtering", in Proc. International Joint Conference on Artificial Intelligence, pp.1019-1023, Kalsruhe: Germany, 1983.

3. KoEnderink, J.J., "The structure of images", Bio. Cybern., 50, pp363-370, 1984.

4. Perona, P. and MaltK, J., "Scale-space and edge detection using anisotropic diffusion", IEEE Trans. Pattern Analysis and Machine Intelligence, 12(7), pp.629-639, 1990.

5. Morel, J-M. And Solimini, S., Variational Methods in Image Segmentation, Birkhäuser: Boston, 1995.

6. Osher, S. and Sethian, J.A., "Fronts propagating with curvature dependent speed: algorithms based on Hamilton-Jacobi formulations", J. Computational Physics, 79, pp.12-49, 1988.

7. Olver, P., Sapiro, G., and Tannenbaum, A., "Invariant geometric evolutions of surfaces and volumetric smoothing", SIAM J. Appl. Math., 57, pp.176-194, 1997.

8. Brakke, K.A., The Motion of a Surface by its Mean Curvature, Princeton Univ. Press, Princeton, NJ, 1978.

9. Gage, M. and Hamilton, R.S., "The heat equation shrinking convex plane curves", J. Differential Geometry, 23, pp.69-96, 1986.

10. Alvarez, L., Lions, P.-L., and Morel, J.-M., "Signal and image restoration using shock filters and anisotropic diffusion", SIAM J. Numerical Analysis, 31(1), 1994.

11. ThIRION, J.P. AND GOURDON, A., "Computing the differential characteristics of isointensity surfaces", Computer Vision and Image Understanding, 61, pp.190-202, 1995.

12. Grenander, U. and Miller, M.I., "Computational Anatomy: An Emerging Discipline", Current and future challenges in the applications of mathematics (Providence, RI, 1997), Quart. Appl. Math., 56(4), pp.617-694, 1998.

13. Gordon, G.G., Face recognition from depth and curvature, PhD Thesis, Harvard University, 1991. 
14. Hallinan, P., Gordon, G., Yuille, A., Giblin, P., and Mumford, D., Two and Three Dimensional Patterns of the Face, A.K.Peters Inc., 1998.

15. Yuille, A.L., "The creation of structure in dynamic shape", Proc. 2nd ICCV, pp.685-689, 1988.

16. Bruce, J.W., Giblin, P.J., And Tari, F., "Parabolic curves of evolving surfaces", IJCV 17(3), pp.291-306, 1996.

17. Bruce, J.W., Giblin, P.J., And TARI, F., "Ridges, crests and sub-parabolic lines of evolving surfaces", IJCV, 18(3), pp.195-210, 1996.

18. Neskovic, P. And Kimia, B., "Three-dimensional shape representation from curvature dependent surface evolution", pp.6-10, ICIP' '95, Austin, Texas.

19. Evans, L.C., "A convergence theorem for solutions of nonlinear second order elliptic equations", Indiana Univ. Math. J., 27, pp.875-887, 1978.

20. Lions, P.-L., "Optimal control of diffusion processes and Hamilton-Jacobi-Bellman equations. I", Comm. Partial Diff. Equations, 8, pp.1134-1101, 1983.

21. Ishit, H., "On uniqueness and existence of viscosity solutions of fully nonlinear second order elliptic PDE's", Comm. Pure Appl. Math., 42, pp.15-45, 1989.

22. Nunziante, D., "Uniqueness of viscosity solutions of fully nonlinear second order parabolic equations with noncontinuous time-dependence", Differential Integral Equations 3, pp.77-91, 1990.

23. Evans, L.C. And Spruck, J., "Motion of level sets by mean curvature. I", J. Differential Geometry, 33, pp.635-681, 1991.

24. ChEN, Y., Giga, Y., AND GOTO, S., "Uniqueness and existence of viscosity solutions of generalized mean curvature flow equations", J. Differential Geometry, 33, pp.749-786, 1991.

25. Kimia, B.B., Tannenbaum, A.R., and Zucker, S.W., "Shapes, shocks, and deformations I: the components of two-dimensional shape and the reaction-diffusion space", $I J C V, 15, \mathrm{pp} .189-$ 224,1995 

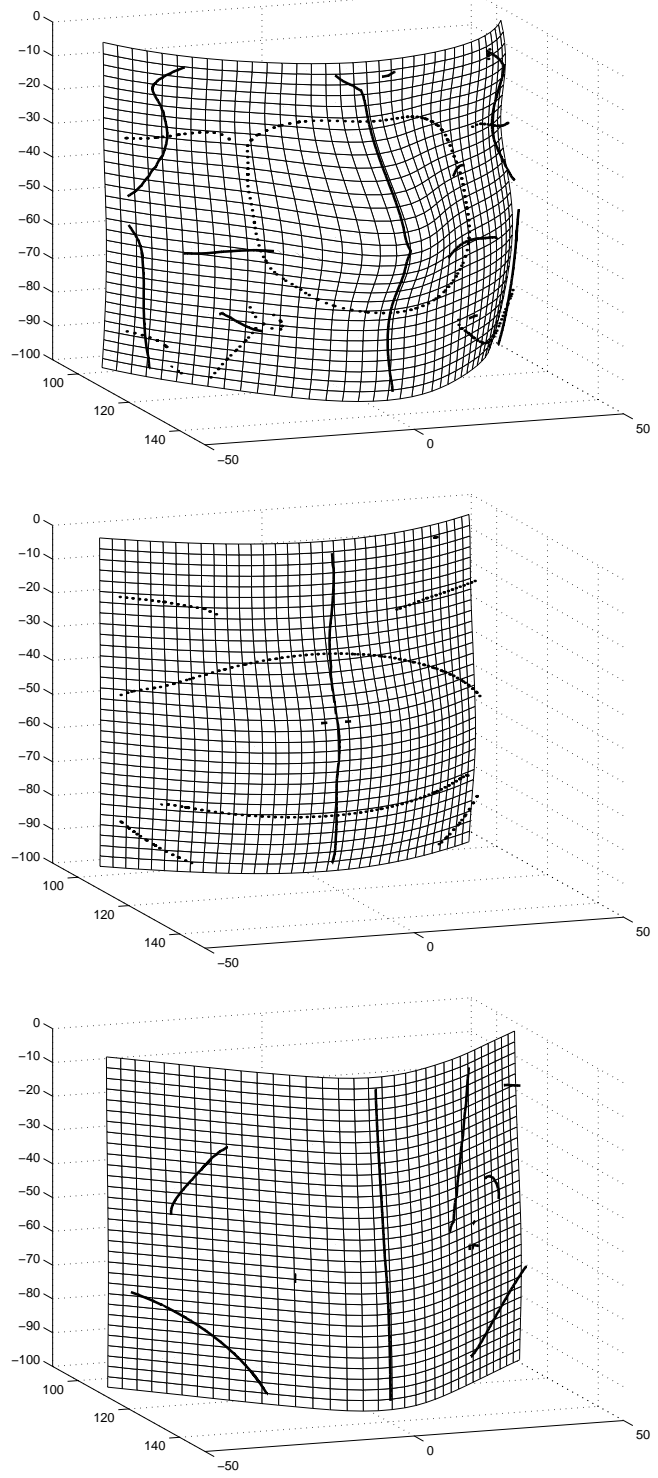

FIG. 1. The 3D face under curvature flows. The first one is the original face, the second and the third ones are the faces under $K_{1}$ and $K_{2}$ flows at $t=1000$ respectively. Blue ridges are shown with solid lines, red ridges with dotted lines. 

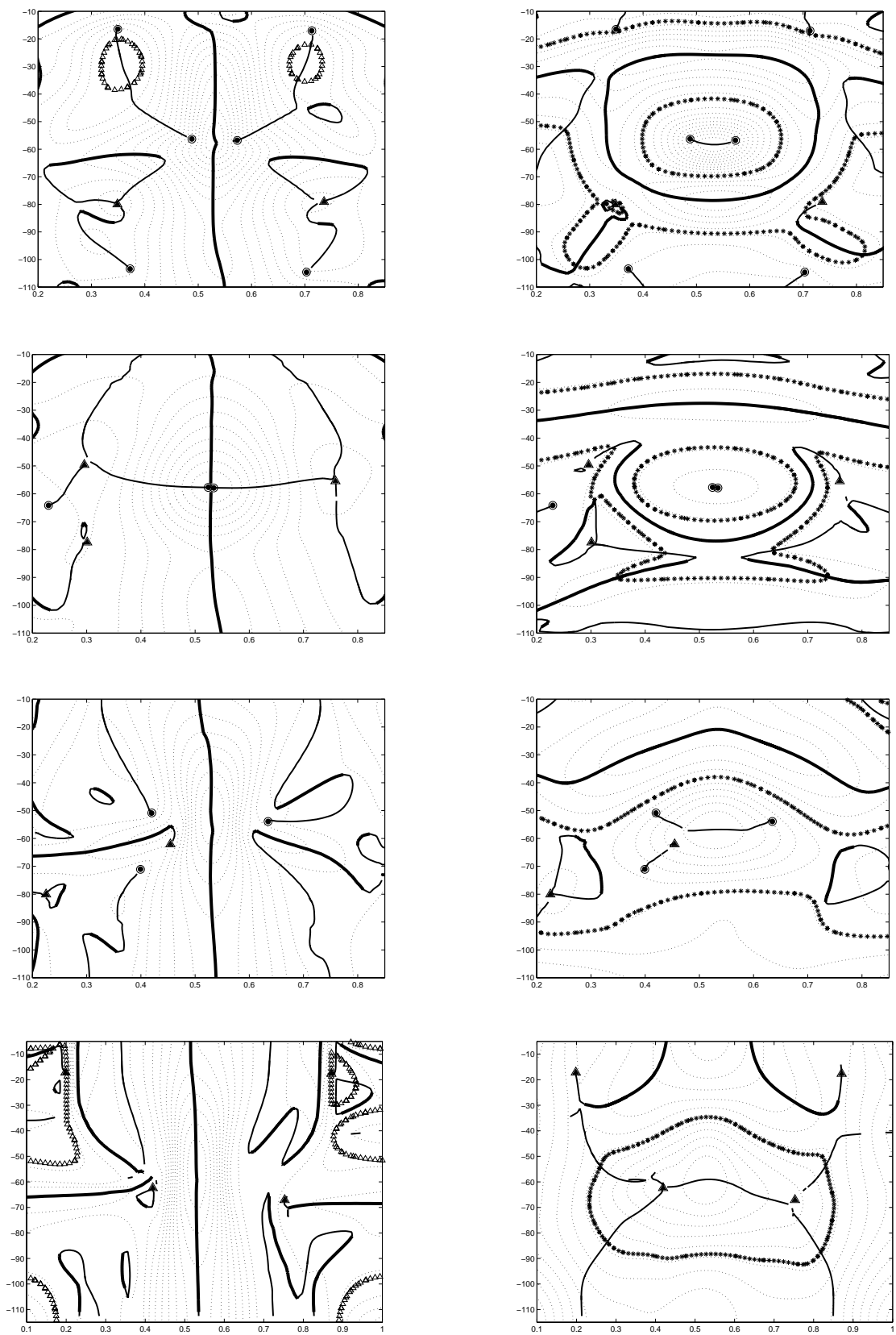

FIG. 2. The evolution of parabolics, ridges and umbilics. The rirst row is the original face, second row is the face under $K_{1}$ flow at $t=100$, third row is the face under $K_{2}$ flow at $t=100$, fourth row is the face under $K_{2}$ flow at $t=250$. On the left, the blue ridges, parabolic lines and the level sets of $K_{1}$ are shown; on the right, the red ridges, parabolic curves and the level sets of $K_{2}$. Thick solid lines are elliptic ridgs, thin grey solid lines are hyperbolic ridges. Trangled curves are blue parabolic curves, starred curves are red parabolic curves. Dotted lines are level sets of curvature. Small solid disks are lemon umbilcs, small solid triangles are star umbilics. 